\title{
Solidification Physics and Microstructure: A Study of AlMg and AIMgSi Alloys by Vortex Method
}

\author{
Socorro Valdez Rodríguez, Lorenzo Martínez and \\ Martín Pech-Canul
}

Additional information is available at the end of the chapter

http://dx.doi.org/10.5772/intechopen.72763

\begin{abstract}
Physical properties on solidification process deal with the internal atomic arrangement, named generally microstructure. The microstructure characteristic is essential for the properties of metallic materials, including binary, ternary or eutectic alloys. Thus, despite significant progress on microstructural evolution, numerous challenges still exist for revealing the internal structure to act synergistically with matrix alloy chemical components. It is well known that the internal microstructure of an alloy controls and modifies the nature, interaction type and properties of the existing defects and final applications. At this point, necessary understanding of the correlation between equilibrium and nonequilibrium effects, surface energy and chemical potential for the time of the structures formation is probably the key to solidification that can help to predict the complex of microstructures. For the case of multi-phase solidification, AlMgSi alloy involves thousands of atoms, at the atomic scale, that transit to microstructures while the solid-liquid phase transformation occurs. This chapter outlines the role of solidification physics, and the atomic arrangement is believed to have in influencing mechanical hardness properties and degradation resistance.
\end{abstract}

Keywords: molecular interaction, microstructure, mechanical properties, mass transport, cooling rate

\section{Introduction}

The purpose of this chapter is to underlie the atomistic theory of AlMg alloy solidification, emphasizing the aspects that relate to microstructure arrangement with the addition of silicon as microalloying. The microstructure characteristic is essential for the properties 
of metallic materials, including binary, ternary or eutectic alloys. This is a problem of pronounced real-world significance as structural materials and also touches on some of the deepest issues of physics like the atomic transport, expressed here in terms that are accessible to a beginner with no background in quantum mechanics. Even though the microstructure considered as a different atomic combination, it reduces to a mass equation [1]. With this in mind, the chapter treaties with the solidification physics, that related the maturity of physically based models of the understanding of AlMg and AlMgSi microstructures. The effect of magnesium as microalloying element has a pronounced solid solubility in aluminum, which develops a high precipitation-hardening capability [2]. The outstanding attributes to AlMg base alloy can increase the motivation to substitute some ferrous alloys with lightweight aluminum-magnesium alloys. Some delicately balanced between experimental and the atomistic theory resulting in an alternative to develop technologies more competitive as production process in order to reduce manufacturing costs and improve material performance.

To understand the importance of aluminum and aluminum alloys, their valuable engineering purposes in automotive parts and aircraft components associated with their lightweight should be mentioned [3-6]. In addition, the aluminum and their alloys possess a good performance, castability, high mechanical strength properties and attractive resistance to electrochemical surface degradation [7-9]. Many investigators describe these advantages as primary kind of light alloys since structural design, architectural applications, to combustion engines, until tribological functions [10-13]. Recently, AlMg alloys have been applied as matrix alloy to composite materials due to their low density and high wettability [14, 15]. Therefore, the development of AlMg alloys is an important task, and the differences between binary, ternary and quaternary alloy make them an important research topic. Interest in the addition of silicon, iron, manganese and silver to the aluminum magnesium matrix is motivated by the marked effect on their corrosion resistance, mechanical behavior and atomic structure arrangement $[16,17]$. Thus, despite significant progress on microstructural evolution, numerous challenges still exist for revealing the internal structure to act synergistically with matrix alloy chemical components. Moreover, the alloying elements, such as silicon, exhibited unique structural arrangement that elevated the mechanical behavior reached by precipitation development occurred after thermal treatment [18]. The precipitation in these alloys indicates a several transformation sequences from the supersaturated solid solution that relies on the fine precipitation, which strained the crystallographic planes of the Al-matrix and improving the specific Young's modulus, high specific strength, yield and creep strengths [19-22]. The silicon and iron microalloying can refine microstructure of AlMg alloys due to the formation of $\mathrm{Mg}_{2} \mathrm{Si}$ and $\mathrm{Al}_{2} \mathrm{MgC}_{2}$ phases during the solidification process $[23,24]$. Interest in the addition of silicon and $\mathrm{Sc}$ to the aluminum magnesium matrix is motivated by the marked effect on their corrosion resistance, mechanical behavior, and atomic structure arrangement $[25,26]$. Moreover, various averaging procedures suggest that AlMgSi alloys reach up to $90 \%$ extruded volume owing to an attractive combination of mechanical and chemical properties and an excellent response to surface finishing operations [27-29]. A gradual increase of extruded pieces continued growth by the 6000 series like in automotive, marine and architectural applications [13, 21]. 
Thus, despite significant progress on microstructural evolution, numerous challenges still exist for revealing the internal structure to act synergistically with matrix alloy chemical components [3]. It is well known that the internal microstructure of an alloy controls and modifies the nature, interaction type and properties of the existing defects and final applications [4, 25].

In general, the physical properties on solidification process deal with the evolution of microstructures during the solid-liquid phase transformation. Additionally, materials science involves the solid-liquid transformation in the manufacturing of metallic alloys, quasicrystal synthesis, composite materials and the new entropic alloy systems [16]. Furthermore, the solidification process has been a key factor in modern technologies for obtaining casting parts that have been based on physical and mechanical treatment applied to alloys in a molten condition $[27,28]$. Therefore, the velocity solidification, related with solute partition, influences the solid-liquid interface atomic diffusion [9]. The internal atomic arrangement, named generally microstructure, as retrieved from the mold, is significant for new materials design and new manufacturing process. It seems that with the appropriate chemical alloying, a relevant material properties could lead to microstructural influences in the various properties including friction and wear, thermal, mechanical, electrical, electrochemical degradation, corrosion resistance, formability and welding characteristics. This chapter outlines the role of solidification physics, and the atomic arrangement is believed to have in influencing mechanical hardness properties and degradation resistance.

\section{Atomistic view of solidification of AlMgSi alloy}

One of its main supposed advantages is the conceptual framework, which underlies the atomistic or microscopic theory of matter that conveys of atoms building micro and nanostructures [14]. In keeping with the research, continues a few practical examples to design engineer materials and devices on an atomic scale as well as to predict the structure of new materials; the flow of current through a nanostructure; their electrical, physical and mechanical properties and the rates of chemical reactions [11-13]. Here, we would like to highlight the microstructural arrangement that makes major contributions in the condensed matter to the development of crystalline and electronic structures correlated with wear life, resistance to reactive degradation, novel electronic devices and nonequilibrium entropic alloys [4, 15]. At this point, necessary understanding of the correlation between equilibrium and non-equilibrium effects, surface energy and chemical potential for the time of the structures formation is probably the key to solidification that can help to predict the complex of microstructures.

The multi-phase solidification of AlMgSi alloy or melting this material for small-scale physical phenomena to incorporate in large-scale models implies a multi-physics nature of solidification [26]. The solidification process involves hundreds of thousands of atoms, related with their melting point, when nucleation initiates from solid-like regions, of the new phase, on the atomic scale until nuclei growths until a crystal during a large fraction of microsecond. In the formation of the solid phase, the surface energy contributes to instability of the liquid/ solid interface [27]. At atomic scale, the conjunction of crystals proceeds in a complementary 
network of grains with heterogeneities like voids, vacancies, dislocations and evidently grain boundaries.

\subsection{AlMgSi phases}

Taking into account our microstructural data material, firstly the AlMg alloy with the phase diagram from Figure 1, their essential phase corresponds to the $\alpha$ and $\beta$ solid phases of $\mathrm{Al}$ and $\mathrm{Mg}$, respectively, and the presence of intermetallic phases. One can observe regions where

only liquid phase $l$ exists,

only solid phase $\alpha$ exists,

liquid $l$ and solid phase $\alpha$ coexist,

liquid $l$ and solid phase $\beta$ coexist,

both solid phases $\alpha$ and $\beta$ coexist.

Taking this last one consideration into account, the growth in that region is also known as eutectic solidification. As we can observe in Figure 2, for AlMgSi alloys phase diagram, intermetallics and grain boundary phases as consequence of thermal treatment process are identified. As one of the principal, intermetallic appears with iron-containing intermetallic and MgSi

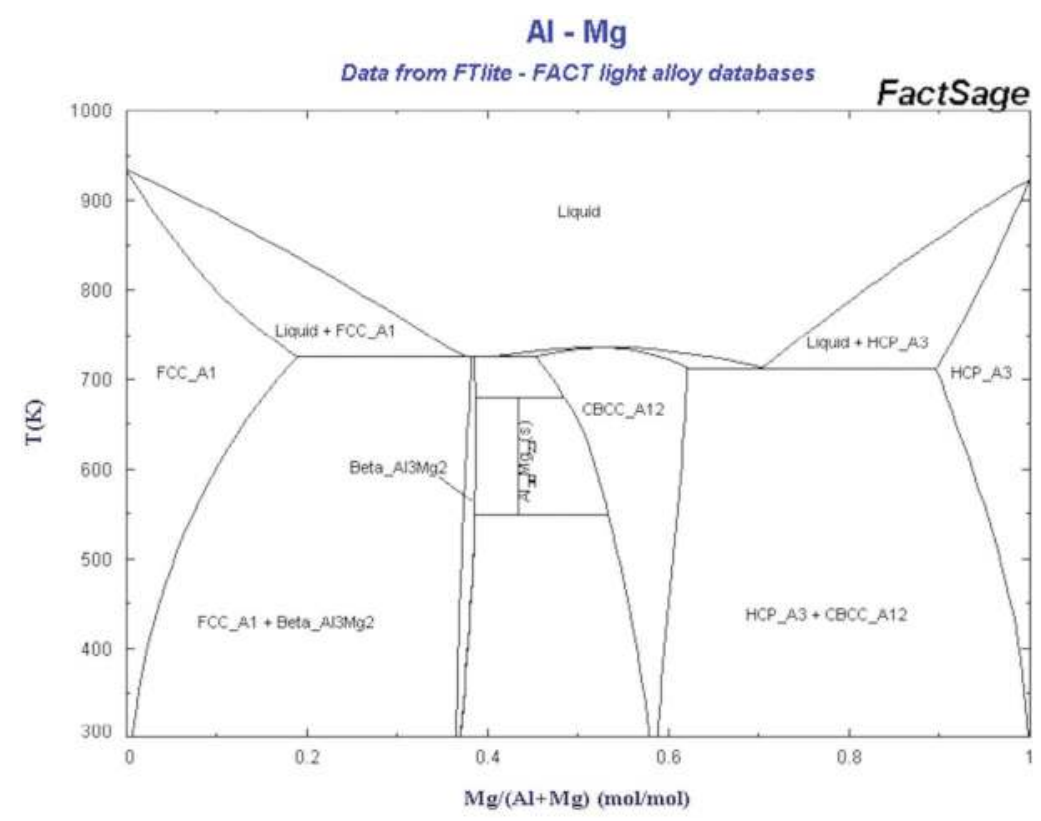

Figure 1. Phase diagram (PD) of binary AlMg alloy [30]. When magnesium in AlMg alloy exceeds its solubility in aluminum liquid, the excessive magnesium will present as intermetallic phases. 


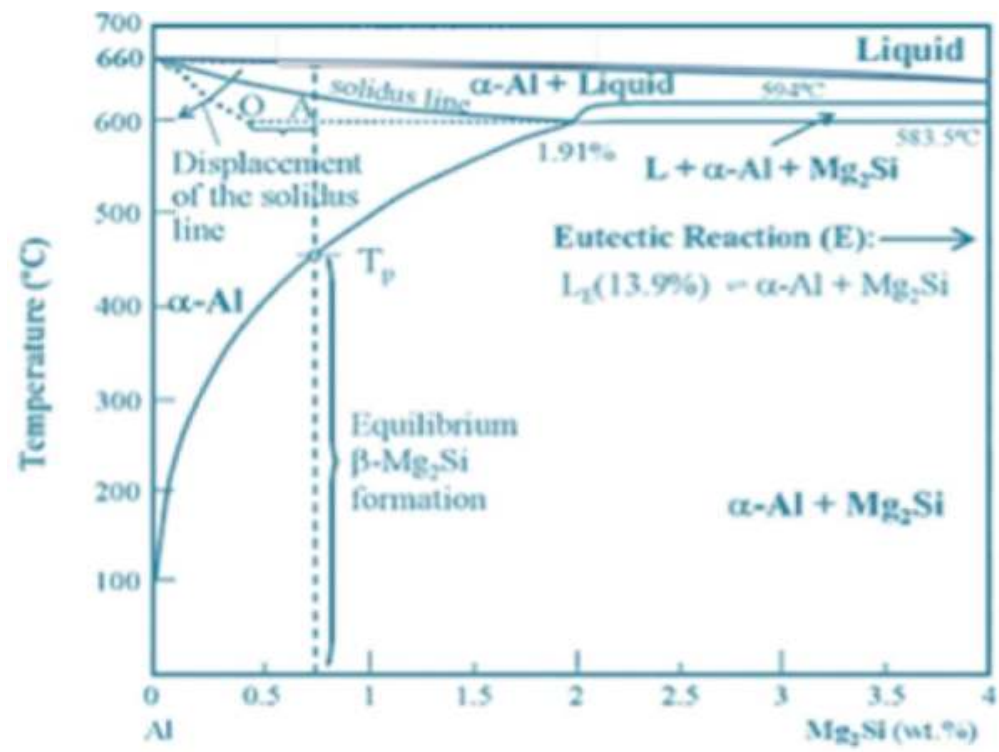

Figure 2. Schematic ternary phase diagram of AlMgSi alloy [31].

precipitates, which possess an ambivalent behavior, by the corrosion process for instance. Alloys on the ternary system $\mathrm{AlMgSi}$ with addition of $\mathrm{Cu}, \mathrm{Fe}, \mathrm{Mn}$ and $\mathrm{Cr}$, among others, possess a precipitation sequence where supersaturated solid solution is rich in non-equilibrium vacancies that enable a passage through a series of metastable precipitates towards the equilibrium, described as SSSS $\rightarrow$ clusters $\rightarrow \beta^{\prime \prime} \rightarrow \beta^{\prime} \rightarrow \beta[5,6]$.

\section{Experimental details}

The 6000 series aluminum alloys containing AlMgSi and AlMgSiCu alloys for such a system are assumed as potential lightweight and high-performance materials to be used in aerospace-craft, aircraft and engine parts in automobiles industry [20]. It has to be accounted for these kinds of systems that the excess of $\mathrm{Si}$ in the simplest case induces a major precipitation of MgSi mainly to obtain higher mechanical strength. With this knowledge in mind, and not forgetting that the most important microstructures of solidifying castings underlies on crystallographic arrangement interconnected with physical, chemical, and nature of the atomic alloying. In the face of these facts, the parameters developed for the solidification sequence of AlMgSi alloy have been the cooling rate, chemical nature and concentrations of microalloying elements. Fundamental considerations were taken throughout the molten AlMg liquid solution in order to vary the contact time between the silicon powder added to the aluminummagnesium molten alloy. 


\subsection{Melting and solidification process details}

Either way, the binary and ternary matrix alloys were processed by stoichiometric ratio of commercially accessible elemental aluminum and magnesium blocks with $99.8 \pm 0.1 \%$ purity and $10.0 \pm 0.02 \mathrm{~mm}^{3}$ size. The heating and melting elements occur inside of alumina crucible kept in a resistance electrical furnace at $720 \pm 5.0^{\circ} \mathrm{C}$. This temperature was associated at overheated of the molten AlMg alloy where an interesting and basic oxidation reaction may appear as well as hydrogen dissolution.

With the purpose that compensates certain oxidation losses, two experimental arrangements were considered. The first one was taken in excess $3 \mathrm{wt} . \% \mathrm{Al}$ and $1.5 \mathrm{wt} . \% \mathrm{Mg}$ to the required quantity of these constituents. The second consideration was a flux mixture of ionic liquid highly concentrated over the crucible to avoid the contact between metallic melt with the environmental atmosphere and the chemical oxidation. Whiskers of silicon carbide with small diameter of $5.0 \pm 0.01 \mathrm{~mm}$ were preheated at $300^{\circ} \mathrm{C}$ and integrated at melt $\mathrm{AlMg}$ binary alloy at $1200 \mathrm{rmp}$ via refractory feeder tip directly into the alumina crucible and then was stirring 5-7 minutes for an improved homogenization. In order to have a major role in control of solidification process, the AlMg alloy, firstly, and the ternary AlMgSi melt, secondly, have been poured into a cylinder-shaped iron mold at $100 \pm 10^{\circ} \mathrm{C}$ and superheated above the aluminum liquidus temperature. Stringent conditions, such as high pressures, high vacuum, well-controlled atmospheres or long fabrication times, are not required. Contrary to previous work, the alloys are manufactured under pressure in a squeeze casting [9] to incorporate ceramic particles into aluminum melt generating a homogenate material. From these data, we can draw that our method resolve difficulties like silicon wetting, sinking or floating and viscosity of molten AlMg alloy added with silicon. In addition, our procedure is low cost and relatively readily available [12]. As a consequence, better mechanical properties, microhardness Vickers (MV) particularly, can be achieved by a wider range of silicon addition [13]. This lead on behalf of different solubility limit, associated at each alloying element that is present in the local distribution.

The nominal chemical composition of tested AlMg and AlMgSi alloys obtained by atomic emission spectrometry with a Spectrolab Lax M8-Windows is given in Table 1. Here, we would like to highlight certain delicately stoichiometric alloy compositions related to this study with regard to the trace or residual levels of certain chemical elements to achieve a desired set of properties in high-performance AlMg and AlMgSi alloys. The most important recent studies deal with the affinity to certain chemical elements like traces of Fe and P to act

\begin{tabular}{lllll}
\hline Material alloy & \multicolumn{2}{l}{ Chemical elements } & \\
\cline { 2 - 5 } & Magnesium & Silicon & Aluminum & Trace elements \\
\hline AlMg binary & $9.58 \pm 0.13$ & $0.11 \pm 0.01$ & Bal. & $0.79 \pm 0.020$ \\
AlMgSi ternary & $9.71 \pm 0.16$ & $2.3 \pm 0.017$ & Bal. & $0.81 \pm 0.011$ \\
\hline
\end{tabular}

Table 1. Chemical composition in wt.\% of AlMg and AlMgSi alloys determined by coupled plasma atomic emission spectroscopy. 
synergistically to the base alloying elements enhancing the effects of basic alloy constituents. For AlMg alloys, $\beta-\mathrm{Mg}_{17} \mathrm{Al}_{12}$ is an essential phase that plays an important role in strengthening crystal boundary. More recent work [28], however, suggests that structural stability mechanism is also explained through the electronic structures. Additional parameter to the trace elements on the alloy phases relates with surface energy in nucleation of the new phase; it is well known that it exerts influence on solubility of components in the binary solutions and contributes to segregation.

\subsection{Metallographic analysis}

Surface metallographic preparation of the casting AlMg and AlMgSi ingots, cooled at conventional solidification velocity, was obtained for revealing the internal structure arrangement of $\mathrm{AlMg}$ and AlMgSi alloys. The physical sectioning at small cylinder specimens was cut with a diamond wheel cutter to gain valuable data about alloys characteristics. Nevertheless, because of the limitation of experimentally detailed solid-liquid interface conditions, the geometry of precision parts close to wall mold produce an accurate model of reality. Due to the surface energy in melts or liquids not varying with direction on the interface, however when the surface of a liquid increased, some atoms come to the surface from the bulk in order to maintain the surface characteristics. For the case of solids, the surface energy differs when the surface is increased, like as the alloys are rolled, as at normal temperatures the atoms mobility is limited [30]. Although the published studies lack data on metallographic and energy surface, this relationship is feasible to resolve the comparative surface energies among crystalline solid phases. From this perspective, the as-cast specimen was carefully encapsulated in epoxy resin, and a basic technique was performed by grinding the samples with SiC from \# 400 to \# 2000 emery papers and mechanically polished with $1 \mu \mathrm{m} \mathrm{Al}_{2} \mathrm{O}_{3}$ power suspension solution in distilled water, followed by etching in Keller's reagent. To prepare the chemical reagent solution, $5 \mathrm{ml}$ of $\mathrm{HNO}_{3}$ was mixed with $3 \mathrm{ml}$ of $\mathrm{HCl}, 2 \mathrm{ml}$ of $\mathrm{HF}$ and $190 \mathrm{ml}$ of distilled water; the etched was done at room temperature for 30-50 s. Chemical reagent acts on surface sample with remarkable morphology definition due to the energy relative to the surface energy. Taking to account what precedes, the relationship energy fluctuates with temperature. For that case, the temperature coefficient of surface energy is associated to the surface entropy, which varies from entropy in the bulk of the metallic material. The surface entropy is commonly positive. This produces the temperature coefficient of surface energy typically negative. Quantitative and qualitative metallography has significant contributions for the physical surface energy variable.

\subsection{Microstructural observations}

The morphologies and arrangement of crystalline phases of the as-cast binary AlMg alloy and ternary AlMgSi alloy samples were examined using scanning electron microscopy (SEM) Jeol 5900 with an accelerating voltage of $25 \mathrm{keV}$. From Leica line-scanning, results of cross section were inspected by electron dispersive spectroscopy (EDS) in order to get the chemical microanalysis and X-ray chemical mapping. A specimen was sectioned at superior, average and inferior part for its chemical and microstructural analyses. These results represent the average of five different analyses in different regions of the surface samples. 
In order to know the crystal structural investigation of AlMg and their alloying, a short wave, monochromatic and collimated beam using copper tube of $K \alpha$ line radiation: $\lambda=0.15406 \mathrm{~nm}$ of X-rays directed on the carefully polished and cleaned surface of the alloys by a Siemens $5000 \mathrm{X}$-ray diffractometer. The angle of incidence, $\theta$, is observed, as $I$ is the intensity of the reflected beam graphite monochromator. The crude results are usually presented as a plot of $I$ vs. $(\sin \theta) / \lambda$, where $\lambda$ is the wavelength of the incident beam recorded from 5 to $120^{\circ}$ with step size 0.02 and $0.6 \mathrm{~s}$ time per step.

\section{Results and discussions}

In order to understand the microstructure evolution of AlMg and AlMgSi alloys, we need to analyze their internal structure throughout the growth of complex dendrite. The dendritic structure is an arrangement of primary and secondary arms, where the cavities between the dendrite branches were occupied by eutectic-type phase. As well as simulations, microstructure [32] reveals that the side branches are created by the development of instabilities induced on the tip of the primary branches. Consequently, the morphology of each phase formed has significant effects on the mechanical and material properties of cast alloys. In addition, provide support for a wide range of structural parameters, we may know the phases and transformations to figure out a new emerging uses that provide the AlMg and AlMgSi alloys, which outperform the competition in demanding automotive applications.

Figure 3 shows the chemical composition by EDS of aluminum, magnesium and silicon in the grain boundary regions, which could be related with the diffusion solutes into the matrix alloy. Hence, the chemical distribution of solute magnesium and silicon is vital for development and verification of atomic solidification process. The most important effect of nature and quantity of alloy elements influences the morphology of a solidification reaction that took place under cooling of a solid transformation; moreover, based on chemical composition, impurities and their influence in the melt nucleation state are very complicated with significantly crystallization conditions.

Analyzing the solidification structure using charge materials, melting process and cooling conditions near to practical foundry situation held a certain time that included the relationship in the liquid-solid transition region, distribution of chemical compositions, identification of metastable system or description for forming temperature and composition solidification microstructures. Throughout the atomic content in each region of phases, the alloy provides the chemical influence on corrosion and mechanical properties. In addition, inoculation and even addition of trace elements are associated at thermodynamics reactions on solid and liquid region, which is reason why the EDS analysis is required. The exact significance of nature and content of magnesium and silicon is reveled in the $\alpha$-Al solid solution with atomic magnesium and silicon dissolved on the gray dark zone. Therefore, this type of alloy obtains solutes like magnesium and silicon distributions associated with the experimental alloy designed and the limit of solubility based on the equilibrium AlMg phase diagram. 

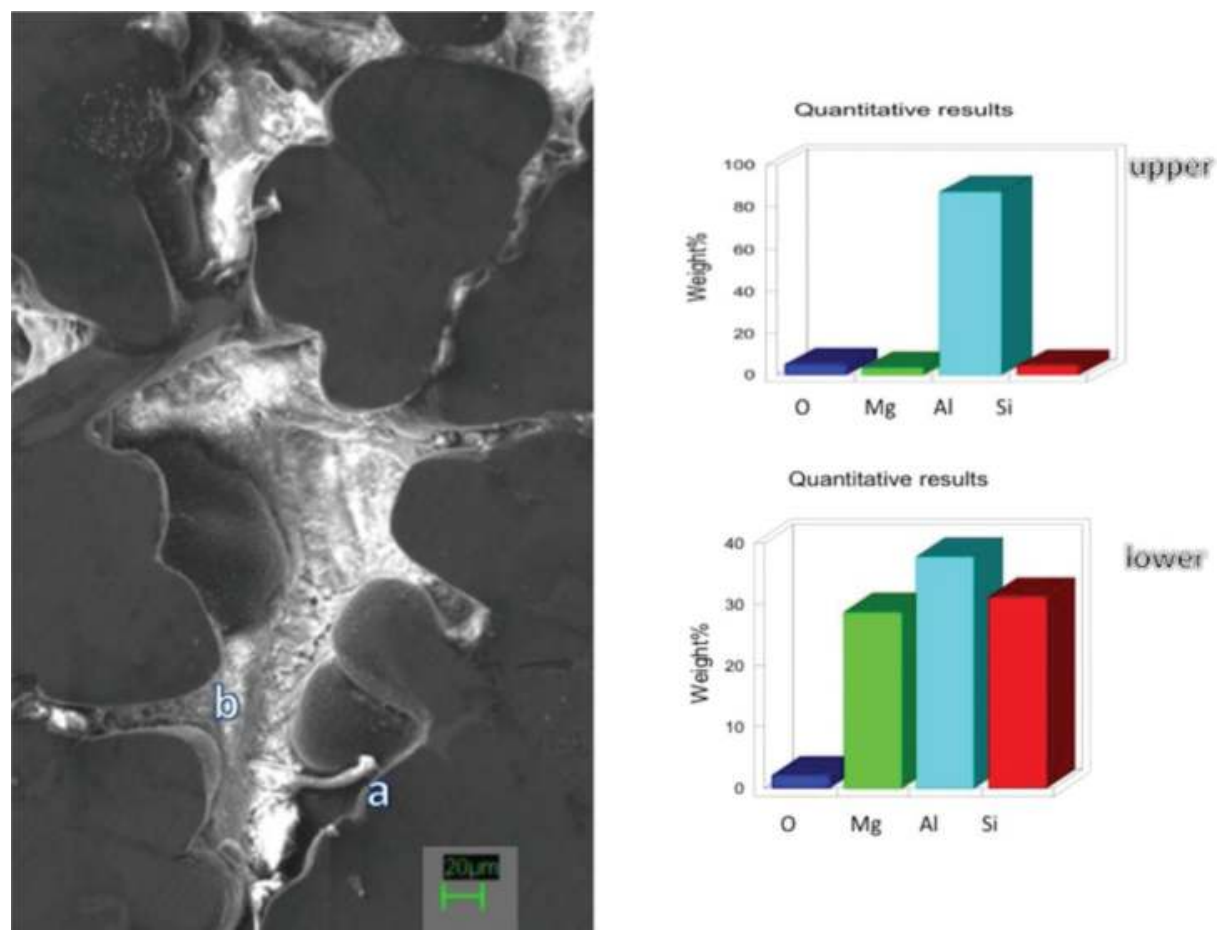

Figure 3. Chemical composition from the AlMg matrix (a) associated with upper graphic bar and interdendritic region (b) that corresponding to lower graphic bar.

The characteristic structural features under the same basic parameters, such as casting temperature and conventional solidification rate, that microstructure features show an equiaxed dendritic morphology observed in a SEM field of back-scattered electrons (BSE) for materials like AlMg and AlMgSi alloys. The microstructure of crystal growth morphology demonstrates the absence of segregation or impurities, and the images of sample surface by secondary electron image (SEI) shown in Figure 4 did not show internal fissuring, associated with abnormal microsegregation. The electron interactions with the AlMg and $\mathrm{AlMgSi}$ atoms produce information about and interdendritic region that can be seen in Figure 4. In past years, many studies have reported that microstructure is strongly related with parameters of solidification route, especially by the solidification rate and chemical nature of alloying. In addition, less distance between the walls of mold increase the cooling rate and modified the structure until fine microstructure and less segregation phases. In addition, Figure 5 shows the crystal structure of AlMg and AlMgSi alloys, which are in agreement with phases reported in equilibrium phase diagram.

In this context, as shown in Figure 3, the images by electron microscopy revealed that magnesium and silicon precipitate to a higher extent of interdendritic region. Generally, a uniform distribution of magnesium in the aluminum matrix is observed in the atomic X-ray mapping. 


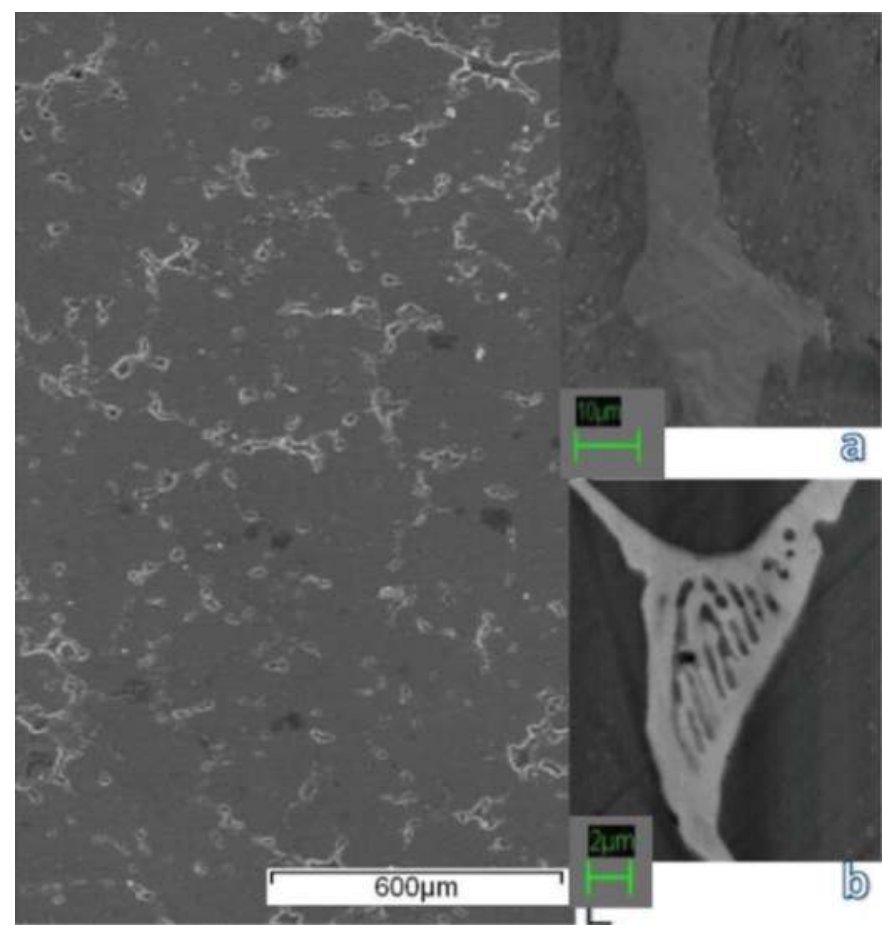

Figure 4. Microstructure of binary AlMg alloy, which was formed by AlMg solid solution (SS) rich in aluminum with dendritic microstructure and revealing the interdendritic morphology.

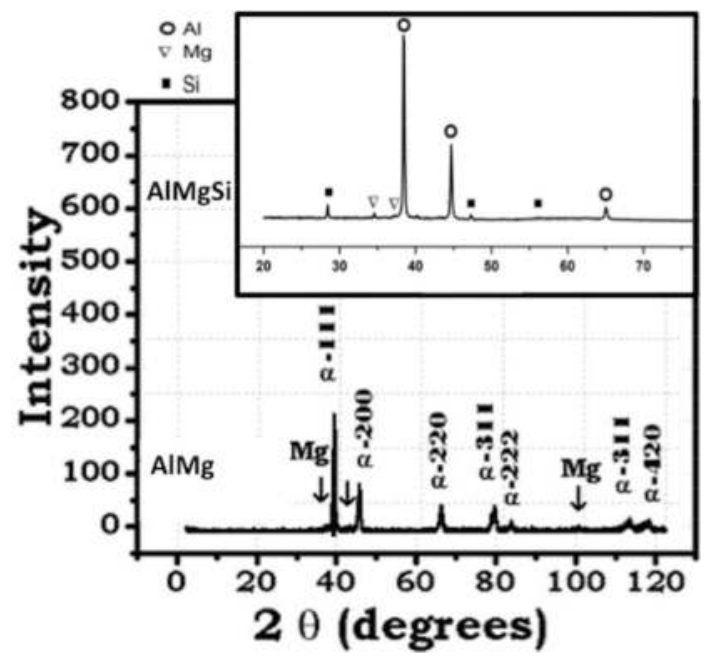

Figure 5. XRD patterns of the AlMg and AlMgSi alloys. 
In contrast, silicon is commonly identified in interdendritic regions as shown in Figure 3. The structure of crystalline solid, from the phase diagram of AlMgSi ternary alloy [21], shows the content of magnesium and silicon as solid solute alloying in the aluminum-rich corner. It seems, therefore, that solutes comprise a solid solution in aluminum solvent matrix at a specific extent. In this respect, the high concentration of magnesium and silicon could made that the diffusion as interchange of atoms in limit of solid solubility produced precipitation of $\mathrm{Mg}_{2} \mathrm{Si}$ phase together with a lower concentration of silicon. However, despite all these metallurgical characterizations, few works concerning the mechanical behavior of these alloys can be found like the research carried out previously [15], where the motion dislocations generated slip in a solid by the motion of dislocations possessing internal strains. However, it should be born in mind that adequate incorporation of grain refinement into the aluminum matrix rise in precipitation due to two physical phenomenal suggestions. The first one deal with rapid heterogeneous nucleation of precipitates, whereas the second one relates an increased rate of dislocation generated near particles added. Characteristically, alloys with precipitates on matrix and refine microstructure age hardening increase the main internal strain and hardness is improved. A further discussion of this fascinating phenomenon of precipitation will be addressed to the corrosion performance, given the fact that the presence of phases along the AlMg matrix may expect a galvanic couple decreasing the corrosion resistance in contact with electrolyte.

\section{Conclusions}

Summarizing the chapter, it may be stated that numerous phenomena assist solidification process.

A brief mention of microstructure influence has been described in relationship with $\mathrm{AlMg}$ and AlMgSi alloys.

The chemical and microstructural details concerning the concentration and distribution of solutes in the unmodified aluminum base alloy were exposed.

Microstructural evidence showed the distribution of phases based on low-cost solidification and basic casting route.

The AlMg and AlMgSi alloys presented Al-rich solid solution matrix with interdendritic regions rich in magnesium and silicon for $\mathrm{AlMgSi}$.

\section{Acknowledgements}

This work was done while visit the Engineering School of BUAP. It is a pleasure to acknowledge the fruitful atmosphere during the visit. This research was supported in part by PAPIITUNAM, acknowledges support from CONACyT. 


\title{
Acronyms and Abbreviations
}

\author{
PD Phase diagram \\ SEM Scanning electron microscopy \\ EDS Electron dispersive spectroscopy \\ MV Microhardness Vickers \\ SS Solid solution \\ XRD X-ray diffraction \\ BSE Back-scattered electrons \\ SEI Secondary electron image
}

\section{Author details}

Socorro Valdez Rodríguez ${ }^{1 *}$, Lorenzo Martínez ${ }^{1}$ and Martín Pech-Canul ${ }^{2}$

*Address all correspondence to: svaldez@fis.unam.mx

1 Institute of Physical Sciences-National Autonomous, University of Mexico ICF-UNAM, University Av. s/n. Cuernavaca, Morelos, México

2 Center for Research and Advanced Studies of the National Polytechnic Institute CinvestavSaltillo, Ramos Arizpe, Coahuila, México

\section{References}

[1] Xu H, Dang Z. Numerical investigation of coupled mass transport and electrochemical reactions in porous SOFC anode microstructure. International Journal of Heat and Mass Transfer. 2017;109:1252-1260. DOI: 10.1016/j.ijheatmasstransfer.2017.02.090

[2] Cuniberti A, Tolley A, Castro Riglos MV, Giovachini R. Influence of natural aging on the precipitation hardening of an AlMgSi alloy. Materials Science and Engineering A. 2010;527:5307-5311. DOI: 10.1016/j.msea.2010.05.003

[3] Valdez S, Bautista A, Villanueva M, Pech-Canul MI. Thermochemistry oxidation of trimetallic alloy modified by thermal procedure. International Journal of Innovative Research in Science, Engineering and Technology. 2016;5:5235-5242. DOI: 10.15680/ IJIRSET.2016.0504131

[4] Valdez S, Campillo B, Pérez R, Martínez L, García A. Synthesis and microstructural characterization of Al-Mg alloy-SiC particle composite. Materials Letters. 2008;62:2623-2625. DOI: 10.1016/j.matlet.2008.01.002 
[5] Gancarz T, Jourdan J, Gasior W, Henein H. Physicochemical properties of Al, Al-Mg and Al-Mg-Zn alloys. Journal of Molecular Liquids. 2018;249:470-476. DOI: 10.1016/j. molliq.2017.11.061

[6] Li H, Cao F, Guo S, Jia Y, Zhang D, Liu Z, Wang P, Scudino S, Sun J. Effects of Mg and $\mathrm{Cu}$ on microstructures and properties of spray-deposited Al-Zn-Mg-Cu alloys. Journal of Alloys and Compounds. 2017;719:89-96. DOI: 10.1016/j.jallcom.2017.05.101

[7] Zhao Z, Sun Z, Liang W, Wang Y, Bian L. Influence of Al and Si additions on the microstructure and mechanical properties of Mg-4Li alloys. Materials Science \& Engineering A. 2017;702:206-217. DOI: 10.1016/j.msea.2017.06.077

[8] Zhang J, Ma M, Shen F, Yi D, Wang B. Influence of deformation and annealing on electrical conductivity, mechanical properties and texture of Al-Mg-Si alloy cables. Materials Science \& Engineering A. 2018;710:27-37. DOI: 10.1016/j.msea.2017.10.065

[9] Kaseem M, Choi K, Ko YG. A highly compact coating responsible for enhancing corrosion properties of Al-Mg-Si alloy. Materials Letters. 2017;196:316-319. DOI: 10.1016/j. matlet.2017.03.106

[10] Lucci R, López Padilla R, Cantero S, Bariles R, Oldani C. Refining of AZ91 magnesium alloy obtained in machining chips recycling. Procedia Materials Science. 2015;8:886-893. DOI: 10.1016/j.mspro.2015.04.149

[11] Chi Y, Gu G, Yu H, Chen C. Laser surface alloying on aluminum and its alloys: A review. Optics and Lasers in Engineering. 2018;100:23-37. DOI: 10.1016/j.optlaseng.2017.07.006

[12] Wan B, Chen W, Lu T, Liu F, Jiang Z, Mao M. Review of solid state recycling of aluminum chips. Resources, Conservation \& Recycling. 2017;125:37-47. DOI: 10.1016/j. resconrec.2017.06.004

[13] Gangil N, Siddiquee AN, Maheshwari S. Aluminium based in-situ composite fabrication through friction stir processing: A review. Journal of Alloys and Compounds. 2017;715:91-104. DOI: 10.1016/j.hallcom.2017.04.309

[14] Anne G, Ramesh MR, Shivananda Nayaka H, Arya SB, Sahu S. Development and characteristics of accumulative roll bonded $\mathrm{Mg}-\mathrm{Zn} / \mathrm{Ce} / \mathrm{Al}$ hybrid composite. Journal of Alloys and Compounds. 2017;724:146-154. DOI: 10.1016/j.jallcom.2017.07.026

[15] Olbricht J, Yawny A, Young ML, Eggeler G. Mechanical and microstructural observations during compression creep of a short fiber reinforced AlMg metal matrix composite. Materials Science and Engineering A. 2009;510-511:407-412. DOI: 10.1016/j.msea. 2008.05.057

[16] Prasad LC, Chatterjee SK, Singh VN. Intermetallic associations in AlMg liquid alloys. Physica B: Condensed Matter. 1996;217:283-291

[17] Ajmera D, Panda E. Thermodynamics of ultra-thin oxide overgrowths on Al-Mg alloys: Role of interface energy. Corrosion Science. 2016;102:425-436. DOI: 10.1016/j.corsci.2015.10.035 
[18] Tanski T, Snopinski P, Pakiela W, Borek W, Prusik K, Rusz S. Structure and properties of AlMg alloy after combination of ECAP and post-ECAP ageing. Archives of Civil and Mechanical Engineering. 2016;16:325-334. DOI: 10.1016/j.a cme.2015.12.004

[19] Umaru OB, Abdulwahab M, Tokan A, Bello AM, Umar HA. Effect of double thermal ageing treatment on the mechanical properties of $\mathrm{Al}-\mathrm{Cu}-\mathrm{Mg} / 3 \%$ rice husk ash composite. Results in Physics. 2016;6:342-345. DOI: 10.1016/j.rinp.2016.06.009

[20] Miyake Y, Sato Y, Teranishi R, Kaneko K. Effect of heat treatments on the microstructure and formability of Al-Mg-Mn-Sc-Zr alloy. Micron. 2017;101:151-155. DOI: 10.1016/j. micron.2017.07.003

[21] Vlach M, Cizek J, Smola B, Melikhova O, Vlcek M, Kodetová V, Kudrnová H, Hruska P. Heat treatment and age hardening of Al-Si-Mg-Mn commercial alloy with addition of Sc and Zr. Materials Characterization. 2017;129:1-8. DOI: 10.1016/j.matchar.2017.04.017

[22] Choi SW, Cho HS, Kang CS, Kumai S. Precipitation dependence of thermal properties for Al-Si-Mg-Cu-(Ti) alloy with various heat treatment. Journal of Alloys and Compounds. 2015;647:1091-1097. DOI: 10.1016/j.jallcom.2015.05.201

[23] Patakham U, Kajornchaiyakul J, Limmaneevichitr C. Grain refinement mechanism in an Al-Si-Mg alloy with scandium. Journal of Alloys and Compounds. 2012;542:177-186. DOI: 10.1016/j.jallcom.2012.07.018

[24] Takai R, Kimura S, Kashiuchi R, Kotaki H, Yoshida M. Grain refinement effects on the strain rate sensitivity and grain boundary sliding in partially solidified Al-5wt $\% \mathrm{Mg}$ alloy. Materials Science \& Engineering A. 2016;667:417-425. DOI: 10.1016/j.msea.2016.05.023

[25] Gariboldi E, Bassani P, Albu M, Hofer F. Presence of silver in the strengthening particles of an Al-Cu-Mg-Si-Zr-Ti-Ag alloy during severe overaging and creep. Acta Materialia. 2017;125:50-57. DOI: 10.1016/j.actamat.2016.11.056

[26] Wang Y, Luo G, Zhang J, Shen Q, Zhang L. Effect of silver interlayer on microstructure and mechanical properties of diffusion-bonded $\mathrm{Mg}$-Al joints. Journal of Alloys and Compounds. 2012;541:458-461. DOI: 10.1016/j.jallcom.2012.06.120

[27] Nanjo F, Kobayashi J, Itoh G. Spinning workability of Al-Mg-Si alloy extruded tube using the forming die. Procedia Engineering. 2017;207:1761-1766. DOI: 10.1016/j.proeng. 2017.10.935

[28] Remoe MS, Marthinsen K, Westermann I, Pedersen K, Royset J, Marioara C. The effect of alloying elements on the ductility of Al-Mg-Si alloys. Materials Science \& Engineering A. 2017;693:60-72. DOI: 10.1016/j.msea.2017.03.078

[29] Razavykia A, Farahany S, Yusof NM. Evaluation of cutting force and surface roughness in the dry turning of $\mathrm{Al}-\mathrm{Mg}_{2} \mathrm{Si}$ in-situ metal matrix composite inoculated with bismuth using DOE approach. Measurement. 2015;76:170-182. DOI: 10.1016/j.measurement.2015.08.032

[30] Valdez S, Chigo-Anota E, Pech Canul MI, Juárez-Islas JA. Degradation analysis of aluminum cellular alloy in saline ionic electrolyte. International Journal of Innovative 
Research in Science, Engineering and Technology. 2015;4:10337-10345. DOI: 10.15680/ IJIRSET.2015.0411004

[31] Voort GV, Suárez-Peña B, Asensio-Lozano J. Microstructure investigations of streak formation in 6063 aluminum extrusions by optical metallographic techniques. Microscopy and Microanalysis. 2013;19:276-284. DOI: 10.1017/S143192761300010X

[32] Liu CL, Azizi-Alizamini H, Parson NC, Poole WJ, Du Q. Transactions of Nonferrous Metals Society of China. 2017;27:747-753. DOI: 10.1016/S1003-6326(17)60085-2 
